\title{
$\mathrm{LV}$.
}

\section{Ueber die Bestimmung der Phosphorsäure mittelst der Oxyde des Urans.}

\author{
Von \\ R. Arendt und Dr. W. Knop.
}

(A. d. chem. Centralblatt 1857. No. 12. von d. Verf. mitgetheilt.)

Wir legen hiermit den Chemikern eine weitere Ausarbeitung der Phosphorsäurebestimmung vor, über welche in dies. Journ. Bd. LXIX, 401 u. f. die ersten Berichte erstattet wurden.

Gegenwärtig, wo wir bereits einige Erfahrungen über Bestimmung der Phosphorsäure durch die Oxyde des Urans gesammelt haben, glauben wir, dass diese Methode in $\mathrm{Zu}-$ kunft nicht ganz unwesentliche Dienste leisten wird. Sie soll die so gute Methode der Bestimmung der Phosphorsäure durch phosphorsaure Ammoniaktalkerde keineswegs verdrängen, sie ist ihr vielmehr gegenüber zu stellen, indem durch dieses letztere Doppelsalz jene Säure aus ammoniakalischen Flüssigkeiten, durch die Uranoxyde aber aus sauren Lösungen ausgefällt werden kann. Dabei sind wir nun allerdings der Ansicht, dass der Umfang einer Methode, die eine Bestimmung der Phosphorsäure in saurer Lösung gestattet, grösser ist, als der der Bestimmung derselben Säure auf die gewöhnliche Art. Thatsächlich haben wir bis jetzt die Genauigkeit der Bestimmung durch die Oxyde des Urans geprüft bei Gegenwart von Kali, Natron, Baryt, Kalk, Talkerde, Eisen, Thonerde, und dadurch die Ueberzeugung gewonnen, dass sie noch auf eine grössere Anzahl von Basen der Formel MO sich ausdehnen lässt.

Bei Gegenwart der Basen von der Formel $\mathrm{M}_{2} \mathrm{O}_{3}$ dagegen können wir diese Art, die Phosphorsäure zu bestimmen, noch nicht als eine wahre quantitative Methode hinstellen, wiewohl wir auch bei diesen zu Approximationen gekommen sind, die nicht ganz verwerflich erscheinen, wie weiter unten bei der Thonerde gezeigt werden soll.

Joura. f. prakt. Chemie. LXX. 7. 
Indessen haben wir auch das Mittel gefunden, einige dieser Oxyde, und darunter gerade dasjenige, von dem man die Phosphorsäure am häufigsten zu trennen hat, nämlich das Eisenoxyd, auf die Oxydationsstufe der Oxydule von der Formel Mo nach einer der ganzen Methode entsprechenden Art und Weise zurückzuführen und am offenen Luftzutritte auf dieser Stufe beliebig lange zu erhalten. Es geschieht dieses durch Uranchlorür, für welches wir eine neue und einfache Darstellung in No. 11. des chemischen Centralblatts, 1857. angegeben haben. Unsere Methode ist deshalb auch zur Scheidung der Phosphorsäure von Eisen anwendbar, nur wird hier die Bedingung gestellt, dass man das Eisen vor Anwendung des essigsauren Uranoxyds durch Uranchlorür in sehr stark saurer Lösung zu Oxydul reducirt habe.

1) Bestimmung der Phosphorsäure bei Gegenwart von Kali, Natron, Talkerde, Kalkerde und Baryt. Bezüglich dieser Bestimmung haben wir den früher gemachten Mittheilungen nur Wenig hinzuzufügen.

Man bewerkstelligt am besten von vorn herein die Lösung mittelst Essigsäure; ist dieses nicht möglich, und hat man einmal die phosphorsauren Basen in Salzsäure oder Salpetersäure gelöst, so versetzt oder fällt man sie durch Ammoniak, bis ein nicht sehr empfindliches Lakmuspapier stark gebläut wird, und löst nun in Essigsäure. Sind jene starken Mineralsäuren zugegen, so soll auch eine nicht geringe Menge essigsaures Ammoniak neben der freien Essigsäure vorhanden sein. Man fügt nun die Lösung von kohlensaurem Uranoxydammoniak in Essigsäure dazu und erhitzt bis zum Kochen, wodurch die Phosphorsäure als gelbes phosphorsaures Uranoxydammoniak ausgeschieden wird.

Einige weitere Bemerkungen hierzu, die wir noch zu machen haben, betreffen nur die mechanische Behandlung des Niederschlages.

Der Niederschlag gehört nämlich zwar nicht zu denen, die die Filterporen verstopfen, er legt sich aber schleimig und dicht an das Filter an. Um ihn auswaschen zu 
können, haben wir zwei verschiedene Wege befolgt, die beide zum Ziele führen.

Der eine ist der von $H$. Rose zur Behandlung des schwefelsauren Baryts (s. Handb. d. analyt. Chemie. 1851. S. 453) empfohlene. Man lässt den Niederschlag nümlich vollständig sich absetzen, decantirt die klare Flüssigkeit so weit als möglich vom Niederschlage auf das Filter, kocht dann den Niederschlag von Neuem mit Wasser, lässt wieder absetzen und decantirt nochmals bis auf den Niederschlag; erst wenn dieser im Becherglase durch Decantiren gewaschen und alles Waschwasser durch das Filter abgelaufen ist, bringt man ihn auf das Filter. Hierbei hat man ihn dann überhaupt nur noch wenig auf dem Filter selbst zu waschen. Das Absetzen geschieht das erste Mal langsam, das zweite, dritte, vierte Mal sehr schnell.

Wenn man die Cohärenz des Niederschlages durch Zusatz eines Körpers aufhebt, der sich in Wasser wie ein Fett verhält, so setzt er sich gleich von vorn herein schnell $a b$ und lässt sich auch leichter auswaschen. Man kann zu diesem Zwecke nicht wohl Fette, Oele und Harzlösungen anwenden, weil sie beim Glühen des Niederschlages das Uranoxyd, vielleicht auch die Phosphorsäure, reduciren. Wir wählten deshalb einen Körper solcher Natur, dass er schon beim Trocknen des Niederschlages vollständig entweichen musste, das Chloroform.

Will man dieses anwenden, so lässt man die Flüssigkeit, nachdem der gelbe Niederschlag darin durch Kochen erzeugt ist, eine kurze Zeit abkühlen und fügt dann 2-3 Tropfen Chloroform dazu. Kann man diese nicht durch Schütteln in dem Niederschlage aufs Feinste vertheilen, so muss es durch ein- bis zweimaliges Aufkochen geschehen. Setzt man die Flüssigkeit nachher bei Seite, so trennt sich der Niederschlag, noch bevor die Flüssigkeit völlig erkaltet ist, ganz scharf von derselben ab und liegt fest am Boden, während die Flüssigkeit selbst vollständig geklärt erscheint. Man kann indessen, wenn man Chloroform angewandt hat, gleich die noch heisse Flüssigkeit a uf das Filter giessen, wenn man dieses sehr gross nimmt 
und mittelst einer Federfahne den Niederschlag öfter aufrührt.

Es ist gut, beim nachherigen Glühen des Niederschlages den Inhalt des Filters vom Filter selbst abzulösen und das Filter erst für sick zu verbrennen. Jedenfalls darf, wenn man mit Salpetersäure befeuchtet, keine Spur von Kohle mehr in der Masse vorhanden sein. Man kann dieses Glühen recht gut im offenen Platinschälchen ausführen, da der Niederschlag nicht schnell Feuchtigkeit anzieht.

Es sei hier noch besonders bemerkt, dass man unter keiner Bedingung einen geglühten Niederschlag nochmals unmittelbar lösen und fällen darf. Die Pyrophosphorsäure verhält sich nicht wie die gewöhnliche. 'Ist durch irgend ein Versehen eine nochmalige Erzeugung des Niederschlages nothwendig, so muss man den geglühten Niederschlag vor dem Lösen in Salpetersäure mit einem grossen Ueberschusse von kohlensaurem Kalinatron schmelzen, um die Pyrophosphorsäure zuverlässig wieder in gewöhnliche zurückzuführen.

2) Trennung der Phosphorsäure vom Eisen. Wie schon in No. 51 des chem. Centralblatts von 1856 erwähnt, schien die Trennung der Phosphorsäure von Eisen mittelst Uranoxyd besondere Schwierigkeiten darzubieten. Neutralisirt man die salzsaure Flüssigkeit, aus welcher die Phosphorsäure abgeschieden werden soll, mit Ammoniak, fügt Essigsäure und dann essigsaures Uranoxyd hinzu, so nimmt, wenn man bis zum Sieden erhitzt, der flockige Niederschlag, der sich anfangs gebildet hatte, eine pulverförmige Beschaffenheit an. Wir erkannten bald, dass derselbe ein eisenhaltiges phosphorsaures Uranoxyd war. Die Menge des darin enthaltenen Eisens ist sehr gering und schwankend; sie hängt, wie weiter unten gezeigt werden wird, davon ab, wie lange man das Sieden fortsetzt. Bei den ersten Bestimmungen der Phosphorsäure in eisenhaltigen Flüssigkeiten war dieses meistens übersehen und der geringe Fehler des Resultates nicht weiter beachtet worden. Spätere Analysen ergaben, dass der Eisengehalt, wenn man, wie angegeben, verfährt, etwa $\mathbf{0 , 5}$ p. C. oder weniger 
beträgt. Ein solcher Niederschlag wurde mit eisenfreiem Cyankalium und kohlensaurem Natron aufgeschlossen, das Uranoxydul abfiltrirt, in Salpetersäure gelöst, durch Ammoniak gefällt und als Oxydoxydul gewogen. Im Filtrate wurde dann das Eisen und die Phosphorsäure auf gewöhnliche Art bestimmt. Die erhaltenen Zahlen sind folgende:

$$
\begin{array}{lr}
\mathrm{U}_{2} \mathrm{O}_{3} & 78,80 \\
\mathrm{Fe}_{2} \mathrm{O}_{3} & \mathbf{0 , 4 6} \\
\mathrm{PO}_{5} & \mathbf{2 0 , 6 5} \\
\hline & 0091
\end{array}
$$

Ist nun auch die Eisenmenge äusserst klein, so konnten wir doch, sollte die Methode an Schärfe anderen nicht nachstehen, dieselbe für Eisen nicht empfehlen, und es war daher unsere Aufgabe, die Bedingungen ausfindig zu machen, unter welchen ein reiner Niederschlag zu erzielen sei.

Vielfach abgeänderte Versuche, die zunächt mit Uranchlorür angestellt wurden, gaben folgende Resultate.

Setzt man zu einer salzsauren Lösung, welche Eisen und Phosphorsäure enthält, Uranchlorürlösung, so bleibt, wenn die Flüssigkeit sauer genug ist, Alles klar; erhitzt man jedoch zum Sieden und verdünnt mit einer grossen Menge heissen Wassers, so scheidet sich ein flockiger, bläulich-grüner Niederschlag von phosphorsaurem Uranoxydul aus. Es ist dieses Salz, wie bereits bekannt, in verdünnter Salzsäure fast unlöslich, und in der That gelingt es, wenn man genug Wasser zusetzt, auf diese Weise alle Phosphorsäure auszufällen. Es giebt für jedes Quantum freie Säure ein Quantum Wasser, welches die Wirkung derselben völlig paralysirt. Der Niederschlag selbst indessen hat keine constante Zusammensetzung. Dazu kommt noch, dass beim Kochen leicht schwarzes Uranoxydul mit niederfällt, was allerdings durch vorher zugefügte sehr grosse Mengen von Salmiak verhütet werden kann. Der Niederschlag enthält je nach Umständen $\mathbf{2 - 3}$ Aeq. Uranoxydul auf 1 Aeq. Phosphorsäure, oder derselbe scheint vielmehr ein wechselndes Gemenge verschiedener phosphorsaurer Oxydulsalze zu sein; eine Formel, wenn diese nicht äusserst gezwungen erscheinen sollte, liess 
sich aus den Analysen fast nie ableiten. Löst man diesen Niederschlag jedoch in Salpetersäure, fügt, um stets sicher zu sein, dass keine Phosphorsäure im Ueberschusse vorhanden ist, Uranoxydlösung hinzu, fällt mit Ammoniak und löst in Essigsäure, so bekommt man in allen den Fällen, wo man die salzsaure Lösung mit der genügenden Menge Wasser versetzt hatte, einen Niederschlag, welcher genau der angewandten Menge Phosphorsäure entspricht. Hauptsächlich scheint die Gegenwart von grösseren Mengen Salmiak dazu beizutragen, genaue Resultate zu erzielen, sei es, dass der ammoniakhaltige Oxydulniederschlag, welcher dadurch entsteht, unlöslicher ist, oder dass überhaupt der Salmiak die an und für sich schon sehr geringe Löslichkeit desselben noch vermindert.

Dies wäre sonach ein Weg, um in dem in Rede stehenden Falle eine Trennung der Phosphorsäure zu bewirken, doch wollen wir denselben durchaus nicht empfehlen. Denn wenn auch die Resultate, sobald man mit genügender Vorsicht arbeitete, stets sehr befriedigend ausfielen, so verkennen wir doch keineswegs, dass die Methode ihre Mängel besitzt. Zunächst würde sie nur da anwendbar sein, wo eben nur die Phosphorsäure bestimmt werden soll. Man erhält unverhältnissmässig grosse Mengen Flüssigkeit und die Bestimmung der übrigen Bestandtheile würde dadurch umständlich gemacht. Ferner ist dazu eine ziemliche Sorgfalt und eine genaue Kenntniss aller dabei vorkommenden Erscheinungen unbedingt erforderlich, wenn man nicht wesentliche Verluste haben will. Es wird nicht leicht gelingen, gleich beim ersten Mal ein exactes Resultat zu erzielen. Aus diesen Gründen beschränken wir uns auf das Mitgetheilte und unterlassen es, Zahlenangaben hinzuzufügen. Wir glaubten jedoch, diese Versuche nicht ganz unerwähnt lassen zu dürfen, wenn auch nur, um Anderen die Mühe zu ersparen, denselben Weg zu betreten.

Alles, was hier bezüglich des Eisens gesagt ist, gilt auch bei Gegenwart von Thonerde, und es lässt sich dieses in die wenigen Worte zusammenfassen, dass das Uranoxyd $\mathrm{U}_{2} \mathrm{O}_{3}$ den Basen von der Formel $\mathrm{M}_{2} \mathrm{O}_{3}$ chemisch zu ähnlich ist, als dass man eine viel bedeutendere Affinität zur 
Phosphorsäure bei ihm voraussetzen dürfte, dass dagegen, wenn das Eisen als Oxydul in der Flüssigkeit vorhanden ist, keine Spur davon in den Uranniederschlag übergeht. Es schwebte uns diese Möglichkeit bereits yon allem Anfange vor, und wir versuchten es zuerst, eine Reduction des Eisens durch bekannte Mittel zu erzielen. Schweflige Säure musste verworfen werden, denn sie wirkt selbst auf den Niederschlag ein; eben so konnte Schwefelwasserstoff nicht genügen. Endlich versuchten wir durch Anwendung von Uranchlorür selbst die Reduction zu bewirken, und der Erfolg davon war ein durchaus befriedigender. Das Eisenoxyd wird durch Zusatz einer Uranchlorürlösung sehr leicht schon bei gewöhnlicher Temperatur und schnell beim Erwärmen in Oxydul verwandelt, und ist vor Oxydation völlig geschützt, so lange noch eine Spur Uranoxydul in der Flüssigkeit ist. Fällt man eine solche Lösung mit Ammoniak, so löst sich (natürlich bei Abwesenheit von Phosphorsäure) Alles klar in Essigsäure, und jedes inzwischen gebildete Theilchen Eisenoxyd wird nachher wieder völlig reducirt.

Dies sind die Grundlagen, auf denen wir nun wiederholte Bestimmungen ausführten.

Von einer titrirten Lösung von phosphorsaurem Natron, welche in 6 C. C. genau 0,070 Phosphorsäure enthielt, wurde jenes oben genannte Volumen pipettirt, mit der 2-8fachen Menge Eisen versetzt, und in gleich zu beschreibender Weise behandelt.

Bei 5 Bestimmungen wurde erhalten:

$$
\begin{array}{llllll}
\left.2 \mathrm{U}_{2} \mathrm{O}_{3}\right), \mathrm{PO}_{5} & 0,348 & 0,348 & 0,354 & \mathbf{0 , 3 5 2} & \mathbf{0 , 3 4 7} \\
\mathrm{PO}_{5} & 0,0698 & \mathbf{0 , 0 6 9 8} & \mathbf{0 , 0 7 0 8} & \mathbf{0 , 0 7 0 4} & \mathbf{0 , 0 6 9 4} \\
& \text { Mittel } & \stackrel{\mathrm{PO}}{\mathrm{P}}=\mathbf{0 , 0 7 0} & &
\end{array}
$$

Eine grössere Menge Niederschlag wurde bereitet, ausgewaschen, bis eine herausgenommene Probe, in kochender Salpetersäure gelöst*), in der erkalteten Lösung

*) Man reagire nie mit Rhodan in eincr warmen und nicht sehr verdünnte Salpetersüure haltenden Flüssigkẹit, denn diese bringt solchenfalls eine ähnliche (vorübergehende) Färbung wie Eisen hervor, was leicht zu Täuschungen Veranlassung geben kann. 
mit Rhodankalium keine Eisenreaction zeigte, darauf getrocknet und in bekannter Weise analysirt.

1,043 Grm. Niederschlag gab:

$$
\begin{aligned}
& \mathrm{U}_{3} \mathrm{O}_{4} \quad 0,818=\mathrm{U}_{2} \mathrm{O}_{3} \quad 0,8334=2 \mathrm{At} \text {. } \\
& 2 \mathrm{MgO}, \mathrm{PO}_{5} \quad 0,326=\mathrm{PO}_{5} \quad \frac{0,2087}{1,0421}=1 \mathrm{At} \text {. }
\end{aligned}
$$

Hiermit ist also bewiesen, dass auch bei Gegenwart von Eisen in essigsaurer Lösung derselbe Niederschlag erhalten wird, welcher stets bei unserer Methode resultirt. Er enthält auf 2 Aeq. Uranoxyd 1 Aeq. Phosphorsäure $=$ 1/5 des ganzen Niederschlages.

Was nun die Methorle selbst anbelangt, um bei Gegenwart von Eisen die Phosphorsäure quantitativ zu bestimmen, so ist sie folgende:

Die dazu nöthigen Flüssigkeiten sind:

a) Eine Uranchlorürlösung (völlig frei von Schwefelwasserstoff), welche nach der in No. 12 d. chem. Centralblatts, 1857 beschriebenen Weise bereitet ist.

b) Eine Lösung von essigsaurem Uranoxydul, erhalten durch Fällung des Chlorürs mit Ammoniak und Wiederauflösen in Essigsäure, am besten in der Wärme.

c) Eine Lösung von essigsaurem Uranoxyd.

Man löst die zu untersuchende Substanz in Salzsäure zu einem möglichst kleinen Volumen (höchstens 50 C. C.), fügt zur klaren Flüssigkeit die Lösung a, bis die Farbe einen deutlichen Stich ins Grüne zeigt und 1 Tropfen Rhodankalium darin keine Röthung mehr hervorbringt. Darauf fällt man mit Ammoniak, bis alle freie Salzsäure neutralisirt ist. Das Erscheinen eines Niederschlages ist kein Zeichen, dass-dieser Punkt erreicht ist; denn schon in mässig saurer Flüssigkeit beginnt das phosphorsaure Uransalz sich auszuscheiden. Es ist stets rathsam, durch ein in die Flüssigkeit geworfenes sehr kleines Stück rothes Lakmuspapier sich von der völligen Sättigung der Säure zu überzeugen. Nun fügt man Lösung c. und freie Essigsäure und, falls man nicht sicher sein sollte, dass noch Uranoxydul in der Flüssigkeit enthalten ist, auch noch einige Tropfen ron b. hinzu und erhitzt zum Sieden. Das Ganze muss deutlich grünlich erscheinen und darf keine 
schmutzige Färbung besitzen, veranlasst durch nicht völlig wieder gelöstes Uranoxydul. Hierauf lässt man vollständig absetzen und decantirt dann erst durchs Filter. Den Niederschlag, von dem man so wenig als irgend möglich auf dasselbe bringt, übergiesst man mit Wasser, fügt ein wenig Salmiak hinzu und kocht auf. Jetzt erfolgt das Absetzen in wenigen Minuten, und wenn man dieselbe Operation noch ein Mal wiederholt, so ist der Niederschlag schon fast ausgewaschen. Durch dieses Verfahren erleichtert man sich die Arbeit bedeutend, indem andernfalls das schwierige Filtriren und langwierige Auswaschen des Niederschlages eine üble Beigabe für die Methode sein würde. Man trocknet und verfährt übrigens, wie bereits früher angegeben.

Will man nun im Filtrate das Eisen etc. bestimmen, s $\sigma$ muss das Uran entfernt werden. Man oxydirt in einer Porcellanschale mit Salpetersäure und neutralisirt vorsichtig mit Ammoniak. Ein sehr geringer Ueberschuss davon ist nicht schädlich; man kocht, bis die Flüssigkeit schwach sauer reagirt, wo dann alles Eisen als basisch essigsaures Salz sich ausscheidet. Ein Rückhalt yon Uran ist ihm durch Behandlung mit kohlensaurem Ammoniak in der Kälte zu entziehen. Im Filtrate fällt man das Uran durch Ammoniak.

Im Verlaufe der Versuche, um die Phosphorsäure von der Thonerde zu trennen, fanden wir, dass es in der That auch gelingen kann, durch blosses Kochen mit essigsaurem Uranoxyd eine Trennung derselben vom Eisen und der Thonerde $\mathrm{zu}$ bewirken. Was das Eisen anbetrifft, so ist schon oben erwähnt, dass ein Niederschlag von phosphorsaurem Eisenoxyd in essigsaurer Lösung, wenn man mit essigsaurem Uranoxyd zum Sieden erhitzt, pulverig wird. Ein grosser Theil des Eisens ist jetzt durch Uran verdrängt, die Flüssigkeit ist von gelöstem essigsauren Eisen blutroth geworden. Kocht man nun aber länger, so verwandelt sich der pulverförmige Niederschlag in den bekannten schleimigen und auf diese Weise ist es möglich, 
auch die letzte Spur von Eisen zu entfernen. Ein viertelstündiges Kochen wird in den meisten Fällen genügen. (Quantitative Bestimmungen sind nicht ausgeführt; sie schienen überflüssig, da eine qualitative Probe den Niederschlag als eisenfrei zeigte und die Zusammensetzung desselben von uns so vielfach untersucht ist, dass wohl kein Zweifel mehr obwalten kann.) Ist man nicht gerade im Besitze einer Uranchlorürlösung, so wäre auch diese Trennungsweise zu empfehlen.

Was die Thonerde anbelangt, so zeigt die in No. $\mathbf{4 9}$ des chem. Centralbl., 1856, aufgeführte Analyse, dass auch hier unter denselben Bedingungen, wie sie so eben beim Eisen besihrieben wurden, eine quantitative Trennung der Phosphorsäure von derselben möglich ist, unsere fortgesetzten Arbeiten aber haben uns belehrt, dass diese Trennung nur dann ein brauchbares Resultat liefert, wenn mån einen sehr bedeutenden Ueberschuss von essigsaurem Uranoxyd anwendet. Durch diese grossen Mengen von Uranoxyd, die man zufügen muss, entstehen andere Unbequemlichkeiten, so dass wir im Ganzen diese Art der Trennung nicht als eine gelungene ansehen können. Die Thonerde hält die Phosphorsäure hartnäckig fest, und in einigen Fällen, wo wcnig Uran zugesetzt worden war, ging das Ganze in eine schleimig-trübe Flüssigkeit über, welche schwer, aber so wie sie war, durchs Filter lief und auf demselben fast nichts zurückliess.

3) Verhalten des Uranchlorürs zur Phosphorsäure bei Gegemoart von Thonerde. Wie bereits angegeben, ist die Thonerde von allen oben aufgezählten Basen die einzige, bei der eine exacte Trennung der Phosphorsäure nicht erreicht werden konnte. Offenbar ist die Affinität derselben zur Phosphorsäure nicht viel geringer, als die des Uranoxyds. Die Affinität der Phosphoreäure zum Uranoxydul ist jedenfalls wieder geringer, als die zum Uranoxyd. Das phosphorsaure Oxydul giebt nämlich bei Behandlung mit Kali die Phosphorsäure viel leichter und rollständiger $a b$, als das Oxyd, und beim Glühen sehr dünner Schichten geht es in Oxydsalz über. In Salzsäure aber ist das phosphorsaure Uranoxydul schwerer löslich, als phosphorsaures 
Uranoxyd, und dieses wieder schwerer löslich, als phosphorsaure Thonerde. Es beruht dieses auf der schwachen Affinität des Uranoxyduls zur Salzsäure, oder richtiger darauf, dass ein Uranoxydulsalz bei Behandlung mit wässriger Salzsäure, namentlich beim Kochen, viel leichter freies Uranoxydul und freie Salzsäure, als Uranchlorür bilden würde, da ja Uranchlorür in verdünnter Lösung in Salzsäure und Uranoxydul zerfällt. Eine Möglichkeit, die Phosphorsäure von der Thonerde zu trennen, war daher noch aus diesem Verhalten denkbar. Da es nun wohl selten vorkommen dürfte, dass man die Thonerde allein, ohne Eisen, von der Phosphorsäure zu trennen hätte, so verfuhren wir wie folgt.

Eisenoxyd und Alaun wurden in concentrirter Salzsäure gelöst und mit $6 \mathrm{C}$. C. einer Lösung von phosphorsaurem Natron versetzt, welche in diesem Volum $\mathbf{0 , 0 7 0}$ Phosphorsäure enthielt. Man fügt einen Ueberschuss von Uranchlorür und eine reichliche Menge Salmiak hinzu und dampft in der Porcellanschale ein, bis die Salzmasse nur noch feucht ist und das Verdunsten durch die sich bildenden Salmiakkrusten erschwert wird.

Man fügt nun zu der noch stark salzsauren Flüssigkeit kochendes Wasser, filtrirt und wäscht aus. Nach dem Auswaschen nimmt man den Niederschlag vom Filter, spült dieses so gut als möglich ab, presst das Filterpapier aus und verbrennt es mit etwas Salpeter und kohlensaurem Natron, löst die Asche mittelst Salpetersäure und fügt die Lösung zu dem vom Filter vorher abgenommenen Niederschlage, oxydirt diesen durch Kochen mit Salpetersäure oder Salzsäure und chlorsaurem Kali, unf fällt mit Ammoniak im Ueberschusse. Hierauf fügt man Essigsüure und einige Tropfen essigsaurer Uranoxydlösung dazu, kocht und verfährt, wie wenn man überhaupt mit essigsaurem Uranoxyd bestimmen wollte.

Bei dieser Behandlung wird in Folge der Unlöslichkeit in Salzsäure das phosphorsaure Uranoxydul ausgeschieden, während die Thonerde und das Eisenoxydul als Chlorverbindung in Lösung bleiben. Bestimmungen, welche mit Quantitäten Alaun von 4-10 Grm. und Eisen von 
0,3 - 0,7 Grm. und einer Lösung von phosphorsaurem Natron ausgeführt wurden, die in 6 C. C. den unter A. angebenen Titre hatte, der auf gewöhnliche Weise mittelst Chlormagnesium-Ammoniak bestimmt war, gaben:

A. I. II. III.

\section{Statt 0,070 Phosphorsäure: $0,065 \quad 0,63 \quad 0,068$}

Im Ganzen betrug also der Fehler constant ein Minus im Werthe von 2-7 Milligrm.

Dieser Fehler ist offenbar für eine analytische Methode zu gross, allein es geht aus diesen Versuchen hervor, dass es die Mühe lohnen wird, noch weiter darüber nachzudenken, wie man zum Ziele gelangen könnte. Die Ursache des Fehlers zu erkennen, ist nicht schwer: offenbar ist es die Menge Uranoxyd, welche durch Reduction des Eisenoxyds zu Oxydul aus dem salzsauren Uranoxydule gebildet wird, die mit der Phosphorsäure ein so constantes Salz bildet, dass es durch salzsaures Uranoxydul nicht zersetzt wird. Das phosphorsaure Uranoxyd ist aber in Salzsäure wenn auch schwer, immerhin löslich.

Versuche, durch Zink oder Kupfer in der eingedampften Flüssigkeit dieses Uranoxyd wieder zu Oxydul zu reduciren, haben, wie sich erwarten liess, kein gutes Resultat gegeben, denn durch das Hinzubringen von noch mehr Körpern zu denen, die man trennen will, kann die Arbeit natürlich nur erschwert werden.

Wir müssen daher hier am Schlusse ausdrücklich hervorheben, dass für diejenigen Metalle, deren Oxyde sich von der Oxydationsstufe $\mathrm{M}_{2} \mathrm{O}_{3}$ durch Uranchlorür auf die niedere Stufe von der Formel MO nicht bringen lassen, unsere Methoden, so weit wir sie bis jetzt ausgearbeitet haben, theils zu umständlich, theils nicht genau genug ausfallen würden, falls man sie praktisch anwenden wollte.

Da nun aber die Bestimmung der Phosphorsäure durch phosphorsaure Ammoniaktalkerde nur dann direct angewendet werden kann, wenn nur Alkalien zugegen, die Erden aber alle ausgeschlossen sind, so glauben wir, dass die hiermit abgehandelte Methode eine noch vorhandene Lücke in der analytischen Chemie ausfüllen wird. 
Sie ist der ihr ähnlichen Bestimmung der Phosphorsäure durch Eisenoxyd jedenfalls vorzuziehen. Später wollen wir einen Gang der Aschenanalyse beschreiben, auf deren Ausführung unsere Methode von vorn herein berechnet war, und dadurch darlegen, dass sie wesentliche Erleichterungen dabei schafft.

Möckern, im März 1857.

\section{LVI.}

\section{Ueber das Vorkommen von Tantalsäure im Columbite von Bodenmais.}

Von

\section{R. Hermann.}

Herr F. Oesten hat meine Angaben über den Tantalsäure-Grehalt des Columbits von Bodenmais geprüft und keine Tantalsäure finden können. Der Grund dieses Misslingens liegt klar vor Augen; denn die von Oesten geprüfte Säure hatte ein spec. Gewicht von nur $\mathbf{5 , 3 8}$, während die Säure, die zu meinen Versuchen diente, ein spec. Gew. von 5,71 hatte. Ich muss daher rathen, zu Versuchen über den Tantalsäure-Gehalt des Columbits von Bodenmais Krystalle mit höherem spec. Gewicht, und nicht solche mit dem niedrigsten spec. Gewicht zu wähien.

Dass übrigens der von mir untersuchte Columbit von Bodenmais Tantalsäure enthielt, geht aus den angegebenen Reactionen der abgeschiedenen Säure deutlich hervor. Ich habe den Versuch ihrer Abscheidung nicht blos ein Mal, sondern mehr als zwanzig Mal mit gleichem Erfolge angestellt und nach und nach über 100 Gran reiner Tantalsäure gesammelt. Eben so musste der von H. Rose benutzte Columbit von Bodenmais Tantalsäure enthalten haben, denn dies geht aus dem hohen spec. Gewicht der aus diesem Minerale ausgeschiedenen Pelopsäure von 6,23 